\title{
The Sun as a MHD generator: application of a new heating mechanism for the coronal loops and closed magnetic structures
}

\author{
D. Tsiklauri
}

Joule Physics Laboratory, Institute for Materials Research, School of Computing, Science and Engineering, University of Salford, Salford, Greater Manchester, M5 4WT, UK

Received 31 March 2005 / Accepted 7 June 2005

\section{ABSTRACT}

We investigate the possibility of heating of the loops and other closed magnetic structures in active regions of the solar corona by the flow of solar wind (plus other flows that may be present) across the magnetic field lines (that are perpendicular to the flow), in a similar manner to a conventional MHD generator. A simple model is formulated which allows us to calculate the typical currents generated by this mechanism. This enables us to make a judgement as to whether the loops in solar active regions can be heated by the proposed mechanism through the associated Ohmic dissipation. It is shown that even if the heating region width is of the order of a few proton Larmor radii (this effectively means that a loop is represented by nano-threads, by analogy with the nano-flare coronal heating mechanism, with width of a few proton Larmor radii) and the plasma flow speed is $50 \mathrm{~km} \mathrm{~s}^{-1}$ then only about $1 \%$ of the heating requirement of coronal loops could be met under this conjecture. Other implications of the model, such as a predicted spatial heat distribution along the loop as well as application of the model to the other places e.g. chromosphere and corotating interaction region structures in the solar wind are discussed.

Key words. Sun: corona - solar wind

\section{Introduction}

The coronal heating problem, the puzzle of what maintains the solar corona 200 times hotter than the photosphere, is one of the main outstanding questions in solar physics (see e.g. Aschwanden (2004) for a recent review on the subject). Aschwanden (2004) categorises theoretical models of coronal heating processes into 5 main groups, according to the main underlying or driving physical processes. These groups are (i) DC (Direct Current) stressing and reconnection models, (ii) AC (Alternating Current) wave heating models, (iii) Acoustic heating, (iv) Chromospheric reconnection, (v) Velocity filtration. The main focus of research in the coronal heating theory has been concentrated on the DC and AC type models.

These two types describe the electro-mechanic coronal response to the photospheric driver that provides the ultimate energy source for heating. It is believed that the perturbations of magnetic field are generated e.g. by the convective motions in the solar convective zone. Then the perturbations propagate through the photosphere to the corona and ultimately constitute the main energy source of coronal heating. These perturbations propagate with the Alfvén speed. The judgement of whether the heating is of the DC or AC type can be made by comparing time-scales of the heating with the Alfvén transit time along a coronal loop: (i) When the photospheric driver, e.g. random motion of magnetic field line footpoints, changes the boundary condition on time scales much longer than the Alfvén transit time along a coronal loop, the loop can adjust to the changing boundary condition in a quasi-static manner. Hence the coronal currents are almost direct ones, which attributes a model to the DC type. (ii) However, when the photospheric driver changes faster than a coronal loop can adjust (e.g., by damping and dissipation of incoming Alfvén waves and fast and slow magnetosonic waves generated due their coupling to Alfvén waves on plasma inhomogeneities), the coronal loop experiences an alternating current, which attributes a model to the AC type.

The DC and AC coronal heating models are subdivided further by possibilities of how the currents are dissipated: either by magnetic reconnection (Sturrock \& Uchida 1981; Parker 1983a,b; Priest et al. 2002; Priest 2003; Priest et al. 2003), Ohmic dissipation via current cascading (Ballegooijen 1986; Galsgaard \& Nordlund 1996), and viscous turbulence (Heyvaerts \& Priest 1992; Einaudi et al. 1996b,a) in the case of DC models, or by Alfvénic resonance, i.e. resonant absorption (Ionson 1978; Davila 1987; Poedts et al. 1989; Ofman et al. 1994, 1995; Erdélyi \& Goossens 1994, 1995, 1996; Vasquez \& Hollweg 2004; Ruderman 1999), phase mixing (Heyvaerts \& Priest 1983; Nocera et al. 1986; Parker 1991; Nakariakov et al. 1997; Ruderman et al. 1998, 1999; DeMoortel et al. 2000; Botha et al. 2000; Tsiklauri et al. 2001; Hood et al. 2002; Tsiklauri \& Nakariakov 2002; Tsiklauri et al. 2002, 2003, 2005a,b), and turbulence (Inverarity \& Priest 1995; Dmitruk et al. 2001) in the case of AC models. 
As an alternative to current dissipation, some heating could also be produced by compressional waves (i.e., by acoustic waves or shocks, Kuperus et al. 1981). It should be mentioned that a problem that models with compressional waves face is that such waves cannot propagate from the photosphere into the corona due to the density gradient. However, interest in these have been recently renewed due to the possibility of generation of fast and slow magnetosonic waves via their coupling to Alfvén waves on plasma inhomogeneities (Nakariakov et al. 1997; Tsiklauri et al. 2001; Tsiklauri \& Nakariakov 2002; Tsiklauri et al. 2002, 2003).

Another approach is adopted by the chromospheric reconnection models: the idea is that chromospheric reconnection through generation of fragmented flux-tubes, strong currents, fast magnetosonic waves and upwards plasma flows can contribute to the coronal heating (Sturrock 1999; Ryutova et al. 2001; Furusawa \& Sakai 2000; Sakai et al. 2000a,b, 2001a,b).

Also a completely different physical mechanism has been proposed, velocity filtration, which is based on the influence of the gravitational potential field in the corona on a postulated non-Maxwellian chromospheric velocity distribution (Scudder 1994).

In this paper we investigate yet another mechanism for the heating of solar coronal loops and other closed magnetic structures in general. We study whether the loops could be heated by the flow of solar wind plasma (plus other flows that may be present) across them by generating currents in a similar manner as a conventional Magnetohydrodynamic (MHD) generator. In Sect. 2 we formulate a simple model which allows us to estimate typical currents generated by this mechanism. We show that if the heating region width is of the order of a few proton Larmor radii and the plasma flow speed is $50 \mathrm{~km} \mathrm{~s}^{-1}$ (which some observations do report), the heating of coronal loops could be only $1 \%$ of the heating requirement under this mechanism. In Sect. 3 we discuss some relevant aspects pertaining to the studied mechanism.

\section{Main consideration}

Similarly to the stressed-induced current cascade models (described e.g. in Aschwanden 2004, p. 366), we try to estimate the energy budget necessary to heat the solar corona. It is assumed by the stressed-induced current cascade models that the random footpoint motion stirs up a potential magnetic field, and hence non-potential fields and related currents are generated. One can estimate the Ohmic (Joule) dissipation associated with these currents and whether it can contribute to the coronal heating. The volumetric heating rate $E_{H}$ requirement for a loop with length $l$ which is heated at both footpoints in the units of

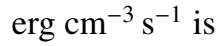

$E_{H 0}=\frac{2 F_{H 0}}{l}=2 \times 10^{-3}\left(\frac{l}{10^{10} \mathrm{~cm}}\right)^{-1}$.

Here we used $F_{H 0}=10^{7} \mathrm{~cm}^{-2} \mathrm{~s}^{-1}$ from Table 9.1 in Aschwanden (2004). The required current density $J$ for the Ohmic dissipation, given by $E_{H}=j^{2} / \sigma$, is hence,

$J=\sqrt{\sigma E_{H 0}}=1.1 \times 10^{7}\left(\frac{l}{10^{10} \mathrm{~cm}}\right)^{-1 / 2} \quad$ (esu).
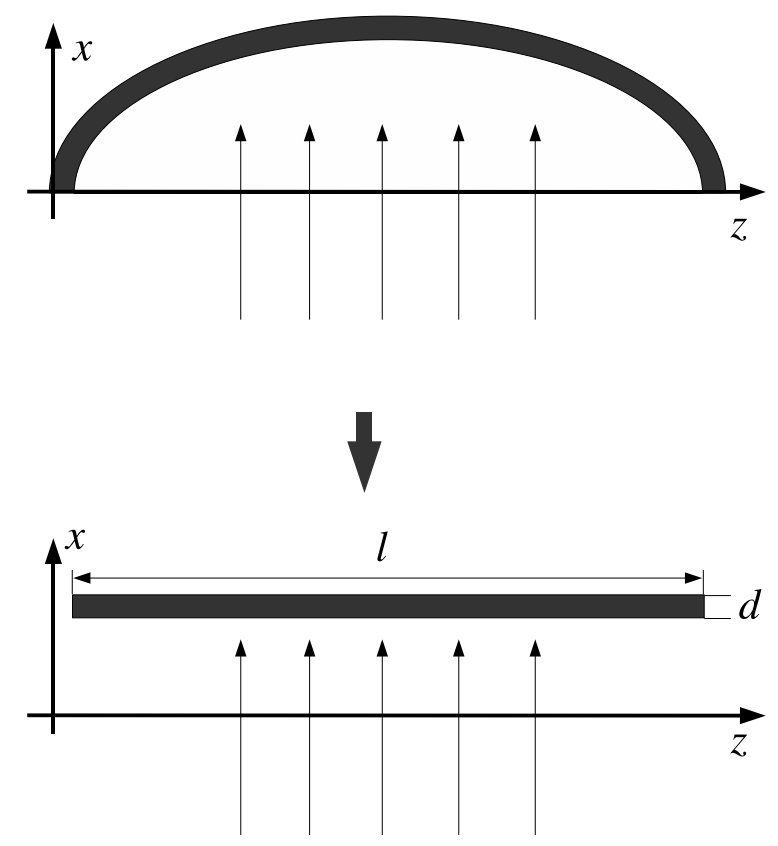

Fig. 1. The sketch of the model.

Here the classical conductivity of $\sigma=6 \times 10^{16} \mathrm{~s}^{-1}$ for a $T=$ $2 \mathrm{MK}$ corona has been assumed.

In general the geometry of a magnetic field in active regions is quite complex. It mostly consists of closed structures such as loops, arcades, canopies, etc. Instead of considering curved magnetic fields, we simplify treatment by approximating the active region loop with a straight slab of length $l(l$ and the associated loop magnetic field are both along the $z$-axis, $\boldsymbol{B}=B z$ ) and radial thickness $d$ ( $d$ is along the $x$-axis). Note that $d$ plays the same role as the length (dimension along the plasma flow) of a MHD generator (Krall \& Trivelpiece 1973). The plasma flows along the $x$-axis with velocity $\boldsymbol{U}=U \boldsymbol{x}$. The sketch of the model is given in Fig. 1. For our model we can write Ohm's law in the following form (e.g. Krall \& Trivelpiece 1973):

$\frac{\boldsymbol{U} \times \boldsymbol{B}}{c}=-\boldsymbol{E}+\frac{\boldsymbol{J}}{\sigma}+\frac{\left|\omega_{\mathrm{ce}}\right| \tau}{B \sigma} \boldsymbol{J} \times \boldsymbol{B}$.

Here we use standard notation with $\boldsymbol{E}, \boldsymbol{J}$ and $\sigma$ being electric field, current density and plasma conductivity respectively. $\omega_{\text {ce }}$ is the electron gyro-frequency and $\tau$ is the time between collisions. Note that we can omit the Hall current, the last term, in the Eq. (3) because we assume that circuit is open in the direction of plasma flow and no current can be sustained. However, current due to the Lorentz force can flow in the $y$-direction (i.e. into the plane of Fig. 1. Note: the generated current flows not along the slab, but across it, in the $y$-direction. This is quite different from the stressed-induced current cascade models (described e.g. in Aschwanden 2004, p. 366) in which azimuthal twisting of the magnetic field lines of a cylindrical loop generates current along the loop. In our model current flows from one edge of the footpoint to the other edge of the same footpoint, but involving the entire slab (which mimics the loop). In a steady state usual MHD equation of motion

$\rho \frac{\partial \boldsymbol{U}}{\partial t}+\rho \boldsymbol{U} \cdot \nabla \boldsymbol{U}=\frac{\boldsymbol{J} \times \boldsymbol{B}}{c}$, 
is reduced to the following form

$\frac{\rho}{2} \frac{\mathrm{d} U^{2}}{\mathrm{~d} x}=\frac{|\boldsymbol{J} \times \boldsymbol{B}|}{c}$.

Note that we neglected the pressure gradient in Eq. (4) because the dominant force in the solar corona is associated with the magnetic field, i.e. plasma $\beta \ll 1$. We can simplify Eq. (5) by putting $\mathrm{d} / \mathrm{d} x \simeq 1 / d$

$\frac{\rho U^{2}}{2} \approx \frac{|\boldsymbol{J} \times \boldsymbol{B}|}{c} d$.

Hence we can estimate the magnitude of the current density $J$ as

$J=\frac{1}{2} \frac{\rho U^{2} c}{B d}$.

Note that the same as Eq. (7) applies to the current density produced by a MHD generator. However, for the latter $d$ is replaced by the length of the MHD generator along which the plasma flows.

Based on Eq. (7) we can make the following estimates for the current density. We use the following typical values (e.g. Aschwanden 2004) of parameters: an electron number density of an active region loop $n_{\mathrm{e}}=2.0 \times 10^{9} \mathrm{~cm}^{-3}$ (hence the density is $\rho=\mu m_{\mathrm{p}} n_{\mathrm{e}}$. We put $\mu=0.61$ corresponding to the totally ionised gas composed of $90 \%$ of hydrogen and $10 \%$ of helium (Sarro et al. 1999)); in closed coronal magnetic structures, Noci et al. (1997), using UVCS operating on board of SOHO, showed that the spatial distribution of the rms velocities of OVI ions, in streamers, is characterised by the fact that in the brightest regions (the streamer core, around the axis, at the lowest heights) the rms velocity is the lowest. The rms velocity is roughly constant in the core while, outside it, it increases, moving both in radial distance and in heliolatitude. Noci et al. (1997) measured rms speeds (in a core of a helmet streamer) to be of the order of $\leq 50 \mathrm{~km} \mathrm{~s}^{-1}$ between $1.5-3 R_{\odot}$. We use this value of $U=50 \mathrm{~km} \mathrm{~s}^{-1}$ in Eq. (7), however this should be used with caution: what is actually measured is the rms speed i.e. some bulk motion of plasma carrying OVI ions with it (through measurement of the OVI $\lambda 1037 / \lambda 1032$ ratio) which may not necessarily be the velocity of the bulk plasma (protons and electrons) across the magnetic field of the closed structures. Some observations (see e.g. bottom right panel of Fig. 4 in Strachan et al. 2002) indicate that outflow speeds of $\mathrm{O}^{5+}$ ions for the two UVCS slit orientations at $2.33 R_{\odot}$ from the Sun's centre i.e. $1.33 R_{\odot}$ above its surface, above a helmet streamer, are less than $\approx 20 \mathrm{~km} \mathrm{~s}^{-1}$ in the central region of the streamer, which is in contradiction with Noci et al. (1997)'s measurements. Although Ofman (2000) argues that outflow speeds for the bulk of the plasma (protons and electrons) should be higher than that for $\mathrm{O}^{5+}$ ions (because the latter drag out through Coulomb collisions), plasma flows still could be as low as $\approx 10 \mathrm{~km} \mathrm{~s}^{-1}$ (Ofman, private communication). Adopting such low values of the plasma outflow speeds would diminish our estimates considerably as the current is proportional to $U^{2}$. For our estimates, we use the more "optimistic" value of $U=50 \mathrm{~km} \mathrm{~s}^{-1}$ quoted above (also see our comment (ii) in the Discussion section). A magnetic field is fixed at 100 Gauss.
Note that we only use longitudinal magnetic field strength, i.e. we ignore azimuthal twist of the magnetic field. There is only evidence for weak twists in coronal loops, of the order of $B_{\phi} / B_{z} \approx 0.01$, from measurements as well as from the kink instability criterion. Thus, taking into account only $B_{z}$ component of the magnetic field seems a good approximation. This is quite different from the stressed-induced current cascade models in which azimuthal twisting of the magnetic field lines of a cylindrical loop generates current along the loop. In our case the current flows from one edge of the footpoint to the other edge of the same footpoint, involving the entire bulk of the slab (that mimics the coronal loop). Additional thought is needed when specifying the parameter $d$. Initially we can use an observed loop width. Aschwanden et al. (2000) present data based on 41 active region loops. They found that typical loop width is a few Mm. Hence putting loop radial thickness $d \approx 1 \mathrm{Mm}$ we obtain

$J=0.08\left(\frac{d}{1 \mathrm{Mm}}\right)^{-1} \quad(\mathrm{esu})$

This estimate is about eight orders of magnitude less than the requirement to heat the solar corona cf. Eq. (2).

In the next estimate, following Aschwanden et al. (2000), we adopt the multi-tread model of a coronal loop. A good graphical representation of this idea can be found in Fig. 1 from Aschwanden et al. (2000), in which a loop is represented by 100 fine loop threads with random footpoint positions and random fluxes. Thus assuming $d=0.01 \mathrm{Mm}$, our estimate of current density will still fall six orders of magnitude less than the coronal heating requirement.

It has been a general trend that as the resolution of EUV telescopes has increased, finer and finer structures were observed. It is generally agreed now that loops have much finer substructure than previously thought. The lowest possible scale for the MHD approximation to be valid is a few Larmor radii of protons, which is $r_{\mathrm{L}}=V_{\perp} / \omega_{\mathrm{cp}}$. Here $\omega_{\mathrm{cp}}=e B / m_{\mathrm{p}} c$ is the proton gyro-frequency. Putting $V_{\perp}=\sqrt{3 k_{\mathrm{B}} T / m_{\mathrm{p}}}$ with a temperature of $T=2 \mathrm{MK}$ and $B=100$ Gauss, $r_{\mathrm{L}}$ is then $23.23 \mathrm{~cm}$ (Shibata \& Tanuma (2001) give a similar estimate for $r_{\mathrm{L}}$ within a factor of $\sqrt{3}$ ). Therefore assuming that a loop consists of substructures, what we call nano-threads (by analogy with the nano-flare coronal heating mechanism), with a width of a few proton Larmor radii for the generated current we obtain

$J=1.1 \times 10^{5}\left(\frac{d}{3 r_{\mathrm{L}}}\right)^{-1} \quad(\mathrm{esu})$

This is only about $1 \%$ of what is required for the heating of solar corona cf. Eq. (2), which obviously cannot be regarded as a sizable contribution to the heating. By obtaining this, one should be also aware of other considerations:

(i) One may question the existence of such nano-treads. There is no direct evidence that the coronal loops consist of threads with width of a few Larmor radii. In fact, essentially all the finest loops observed with TRACE have a width of the order of 1000-2000 km and have a monolithic structure, that is a single temperature at any cross-section, which presently does not support the nano-thread concept. The TRACE CCD camera has 0.5 arcsec pixels which is $366 \mathrm{~km}$ on the sun. Hence it 
is possible that the smallest observed $1000 \mathrm{~km}$ wide monolithic structures are simply too close to the resolution limit and future high spatial resolution space missions may be needed to reveal further sub-structuring, ultimately corroborating or disproving our nano-thread conjecture.

The situation here is possibly similar to the stressedinduced current cascade model, which is based on the random footpoint motion (by twisting the field lines) that predicts a current density, which is about 5 orders of magnitude smaller than that required to satisfy the coronal heating requirement Eq. (2). Aschwanden (2004) concludes that the Joule dissipation is generally inefficient in the corona, unless $10^{5}$ smaller transverse length scales can be produced. This was the main motivation of the model by Ballegooijen (1986), who proposed a current cascade model, where free magnetic energy is transferred from large to small length scales in the corona as a result of the random motion of photospheric footpoints.

(ii) The other mechanisms reviewed in the introduction are the main contributors to the heating budget. While our MHD generator mechanism contributes only $1 \%$ even at the smallest possible scale.

\section{Discussion}

The main novelty of this study is the exploration of a conjecture that loops in the solar corona could be heated by currents which are generated by solar wind (plus other flows that may be present) across the magnetic field lines in a similar fashion as a conventional MHD generator. In previous sections we gave estimates of generated currents and appropriate heating scales.

(i) Spatial distribution of heat deposition along the loop holds the key to an understanding of the physical precesses that are responsible for the heating of the solar corona in general. This quantity can be inferred e.g. from high resolution images produced by the Yohkoh Soft X-Ray Telescope, the SOHO Extreme-Ultraviolet Imaging Telescope and the Transition Region and Coronal Explorer (TRACE). Aschwanden et al. (2000) established that long loops $>100 \mathrm{Mm}$ are preferentially heated near footpoints with the heating scale height of about $20 \mathrm{Mm}$ and are far from hydrostatic equilibrium. Shorter loops $\simeq 20 \mathrm{Mm}$ are heated almost uniformly and are predominantly in hydrostatic equilibrium. Although the heating budget requirement is not met by our model, it predicts that loops with weak curvature (where the radius of curvature is much greater than loop length, $R_{\text {curv }} \gg l$ ) will be heated almost uniformly, as most of the loop (with its magnetic field) will be at $90^{\circ}$ to the plasma flow. Thus, the $\boldsymbol{U} \times \boldsymbol{B}$ term will be significant in all parts of the loop. This is what is seen in the Aschwanden et al. (2000) data: e.g. if we look at images of loops 2, 3, 4 (length $\simeq$ $20 \mathrm{Mm}$ ) of the TRACE $171+195$ data they all have large curvature radii (are almost flat) and are heated uniformly, i.e. in accord with our model prediction. However, as we estimated above the heating requirement is still not met by our model. Hence, the discussion about the spatial distribution of heat deposition along the loop is only of an academic interest. For the loops with strong curvature (radius of curvature is of the order of loop length, $R_{\text {curv }} \simeq l$ ) our model predicts that footpoint parts will not be heated as the magnetic field is almost parallel to the plasma flow and thus at the footpoints the $\boldsymbol{U} \times \boldsymbol{B}$ term will be small and only the apex part of the loop will be heated. If we look at images of loops 30, 31, 33 (length $\simeq 200 \mathrm{Mm}$ ) of the TRACE $171+195$ data they all have curvature radii comparable to the loop length (are quite curved) and are heated near footpoints. This adds an additional problem to our model which predicts no heating at footpoints and heating concentrated at the apex for the reasons explained above. Thus, loops with strong curvature are probably heated by some other mechanism (near the footpoints). However, as for the coronal heating problem itself this does not pose a serious problem as the number of short $(\simeq 20 \mathrm{Mm})$ and flat loops is more significant than the long $(>200 \mathrm{Mm})$ and curved loops, which are more rare.

(ii) For the value of density used in our estimates of the magnitudes of currents generated by our mechanism, we used the observational value of $n_{\mathrm{e}}=2.0 \times 10^{9} \mathrm{~cm}^{-3}$. In a conventional MHD generator the value of density is prescribed by the strength of the magnetic field across the flow (which tends to resist the flow) and the strength of the gradient of the plasma flow through the generator. In the case of coronal loops however there are also up-flows of plasma from the chromosphere and transition region. Svestka et al. (1998) suggested that in active regions there are loop systems that are embedded in hot coronal structures looking in soft X-rays like fans of coronal rays. These structures are formed during the flare and extend high into the corona. Svestka et al. (1998) found that such structures create mass flow from the active region into interplanetary space and these are the sources of a density enhancement in the solar wind. This suggestion is supported by synoptic maps of solar wind sources constructed from scintillation measurements which show a source of enhanced solar wind density at the position of the active region. It is not entirely clear at this stage what effect the above complex factors could have on our simple calculation, but we believe in the overall correctness of our model and its predictions. At any rate one can conjecture that the observed density in the active regions of $n_{\mathrm{e}}=2.0 \times 10^{9} \mathrm{~cm}^{-3}$ is caused by the fact that the closed magnetic field lines, transverse to the plasma flow, resist the flow, making it dense, such that the observed density is obtained. The same occurs in an MHD generator.

(iii) The difference between our model and the $\mathrm{AC}$ and DC models mentioned in the introduction is as following. For the latter two the main source of energy is the convective motions that drive either reconnection events or generate waves which then dissipate. In our case, however, the source of energy is in the plasma flow across the magnetic field lines, i.e. solar wind plus other flows that may be present. Commenting on possibility of actual flow across the magnetic field in the coronal conditions we would like to stress that the parameter that controls the possibility is $\sigma$, the plasma conductivity. Since we do not use any enhanced values for $\sigma$ and the bulk flows in the cores of helmet streamers are observed (Noci et al. 1997), we believe that flows across the magnetic field are possible. Note that the smallness of plasma- $\beta$ in the corona is not a problem for our model as this parameter controls only compressibility of plasma, not the cross-field flow.

(iv) We would like to point out a possibility to apply our mechanism in the chromospheric footpoints of the loops 
and in the corotating interaction region structures of the solar wind. For example, in the chromosphere the neutrals are abundant and the frozen-in condition which inhibits the cross-field plasma flow can perhaps be relaxed. Possible across-the-field flows are Evershed flows in sunspots. Also, various granulation motions can be transverse to the field and hence produce the current. Although in the chromospheric footpoints there are only very small random motions detected in the horizontal direction (and thus perpendicular to the vertical magnetic field), of the order of $10 \mathrm{~km} \mathrm{~s}^{-1}$, which yield currents four orders of magnitude below the coronal heating requirement. But density in the chromosphere is much higher, hence the value of the generated current could be enhanced by orders of magnitude. Also, further out in the corotating interaction region structures (Gosling 1996), in the solar wind, the magnetic field has a component transverse to the plasma flow and hence currents can be generated.

We also would like to estimate how "efficient" the Sun is as MHD generator: the dimensionless ratio which quantifies this is $N=\sigma B^{2} d /\left(\rho U c^{2}\right)$ (in CGS units). For the most "optimistic" estimate (Eq. (9)) along with the classical conductivity of $\sigma=6 \times 10^{16} \mathrm{~s}^{-1}$ for a $T=2 \mathrm{MK}$ corona, $N=4.6 \times 10^{9} \gg 1$. This means that, on the one hand, the Sun appears to be quite efficient as MHD generator, as for typical ionised gas MHD generators $N \approx 10$ (Heywood 1968). On the other hand, despite this, the coronal heating budget is still not met by our mechanism.

Interestingly, our conjecture of the MHD generator can perhaps lead to yet another interesting possibility: if instead of generating current across the loop (not along the loop) via the flow of plasma across the magnetic field, an electric field (created e.g. by some reconnection type event) can be applied across the $y$-coordinate into the plane of Fig. 1 i.e. from one edge of the footpoint to the other edge of the same footpoint, involving the entire bulk of the slab (that mimics the coronal loop), then the Lorentz force reverses its direction (see Fig. 3 in Heywood 1968) and the system can act as MHD pump, i.e. accelerate the plasma flow. Whether this is of any relevance to the solar wind acceleration remains to be established.

Acknowledgements. The author kindly acknowledges support from Nuffield Foundation through an award to newly appointed lecturers in Science, Engineering and Mathematics (NUF-NAL 04). The author would like to thank L. Ofman and V. Nakariakov for critical comments; L. Strachan for the additional data from his paper; the Editor, W. Schmidt, for useful comments, and B. Roberts for the encouragement and good advice.

\section{References}

Aschwanden, M. J. 2004, Physics of the solar corona an introduction (Chichester, UK: Praxis Publishing Ltd)

Aschwanden, M. J., Nightingale, R. W., \& Alexander, D. 2000, ApJ, 541, 1059

Ballegooijen, A. A. V. 1986, ApJ, 311, 1001

Botha, G. J. J., Arber, T. D., Nakariakov, V. M., \& Keenan, F. P. 2000, A\&A, 363, 1186

Davila, J. M. 1987, ApJ, 317, 514

DeMoortel, I., Hood, A. W., \& Arber, T. D. 2000, A\&A, 354, 334
Dmitruk, P., Milano, L. J., \& Matthaeus, W. H. 2001, ApJ, 548, 482

Einaudi, G., Califano, F., \& Chiuderi, C. 1996a, ApJ, 472, 853

Einaudi, G., Velli, M., Politano, H., et al. 1996b, ApJ, 457, L113

Erdélyi, R., \& Goossens, M. 1994, Ap\&SS, 213, 273

Erdélyi, R., \& Goossens, M. 1995, Sol. Phys., 294, 575

Erdélyi, R., \& Goossens, M. 1996, A\&A, 313, 664

Furusawa, K., \& Sakai, J. I. 2000, ApJ, 540, 1156

Galsgaard, K., \& Nordlund, A. 1996, J. Geophys. Res., 101, 13445

Gosling, J. T. 1996, ARA\&A, 34, 35

Heyvaerts, J., \& Priest, E. R. 1983, A\&A, 117, 220

Heyvaerts, J., \& Priest, E. R. 1992, ApJ, 390, 297

Heywood, J. B. 1968, Phys. Edu., 3, 260

Hood, A. W., Brooks, S. J., \& Wright, A. N. 2002, Proc. Roy. Soc. Lond. A, 458, 2307

Inverarity, G. W., \& Priest, E. R. 1995, A\&A, 302, 567

Ionson, J. A. 1978, ApJ, 226, 650

Krall, N. A., \& Trivelpiece, A. W. 1973, Principles of Plasma Physics (New York: McGraw-Hill)

Kuperus, M., Ionson, J. A., \& Spicer, D. 1981, ARA\&A, 19, 7

Nakariakov, V. M., Roberts, B., \& Murawski, K. 1997, Sol. Phys., 175, 93

Nocera, L., Priest, E. R., \& Hollweg, J. V. 1986, Geophys. Astrophys. Fl. Dyn., 35, 111

Noci, G., Kohl, J. L., Antonucci, E., et al. 1997, in Proc. SOHO 5, ed. A. Wilson (ESA SP-404; Noordwijk: ESA), 75

Ofman, L. 2000, Geophys. Res. Lett., 27, 2885

Ofman, L., Davila, J. M., \& Steinolfson, R. S. 1994, ApJ, 421, 360

Ofman, L., Davila, J. M., \& Steinolfson, R. S. 1995, ApJ, 444, 471

Parker, E. N. 1983a, ApJ, 264, 642

Parker, E. N. 1983b, ApJ, 330, 474

Parker, E. N. 1991, ApJ, 376, 355

Poedts, S., Goossens, M., \& Kerner, W. 1989, Sol. Phys., 123, 83

Priest, E. R. 2003, Adv. Space Res., 32, 1021

Priest, E. R., Heyvaerts, J. F., \& Title, A. M. 2002, ApJ, 576, 533

Priest, E. R., Longcope, D. W., \& Titov, V. S. 2003, ApJ, 598, 667

Ruderman, M. S. 1999, ApJ, 521, 851

Ruderman, M.S., Nakariakov, V. M., \& Roberts, B. 1998, A\&A, 338, 1118

Ruderman, M. S., Goldstein, M. L., Roberts, D. A., et al. 1999, J. Geophys. Res., 104, 17057

Ryutova, M., Habbal, S., Woo, R., et al. 2001, Sol. Phys., 200, 213

Sakai, J. I., Kawata, T., Yoshida, K., et al. 2000a, ApJ, 537, 1063

Sakai, J. I., Mizuhata, Y., Kawata, T., et al. 2000b, ApJ, 544, 1108

Sakai, J. I., Minamizuka, R., Kawata, T., et al. 2001a, ApJ, 550, 1075

Sakai, J. I., Takahata, A., \& Sokolov, I. V. 2001b, ApJ, 556, 905

Sarro, L. M., Erdélyi, R., Doyle, J. G., et al. 1999, A\&A, 351, 721

Scudder, J. D. 1994, ApJ, 427, 446

Shibata, K., \& Tanuma, S. 2001, Ear. Plan. Sp., 53, 473

Strachan, L., Suleiman, R., Panasyuk, A. V., et al. 2002, ApJ, 571, 1008

Sturrock, P. A. 1999, ApJ, 521, 451

Sturrock, P. A., \& Uchida, Y. 1981, ApJ, 246, 331

Svestka, Z., Farnik, F., Hudson, H. S., et al. 1998, Sol. Phys., 182, 179

Tsiklauri, D., \& Nakariakov, V. M. 2002, A\&A, 393, 321

Tsiklauri, D., Arber, T. D., \& Nakariakov, V. M. 2001, A\&A, 379, 1098

Tsiklauri, D., Nakariakov, V. M., \& Arber, T. D. 2002, A\&A, 395, 285

Tsiklauri, D., Nakariakov, V. M., \& Rowlands, G. 2003, A\&A, 400, 1051

Tsiklauri, D., Sakai, J. I., \& Saito, S. 2005a, New J. Phys., 7, 79

Tsiklauri, D., Sakai, J. I., \& Saito, S. 2005b, A\&A, 435, 1105

Vasquez, B. J., \& Hollweg, J. V. 2004, Geophys. Res. Lett., 31, L14803 